\title{
Amplification and attenuation of a probe signal by doubly-dressed states
}

\author{
S. N. Shevchenko*, ${ }^{1,2}$ G. Oelsner* ${ }^{3}$ Ya. S. Greenberg, ${ }^{4}$ P. Macha, ${ }^{3}$ D. S. \\ Karpov, ${ }^{1,2}$ M. Grajcar, ${ }^{5}$ U. Hübner, ${ }^{3}$ A. N. Omelyanchouk, ${ }^{1}$ and E. Il'ichev ${ }^{3}$ \\ ${ }^{I}$ B. Verkin Institute for Low Temperature Physics and Engineering, Kharkov, Ukraine \\ ${ }^{2}$ V. Karazin Kharkov National University, Kharkov, Ukraine \\ ${ }^{3}$ Leibniz Institute of Photonic Technology, Jena, Germany \\ ${ }^{4}$ Novosibirsk State Technical University, Novosibirsk, Russia \\ ${ }^{5}$ Department of Experimental Physics, Comenius University, Bratislava, Slovakia
}

(Dated: February 2, 2018)

\begin{abstract}
We analyze a system composed of a qubit coupled to the electromagnetic fields in two high quality quantum oscillators. A particular realization of such a system is the superconducting qubit coupled to a transmission-line resonator driven by two signals with frequencies close to the resonator's harmonics. This doubly-driven system can be described in terms of the doubly-dressed qubit states. Our calculations demonstrate the possibility to change the number of photons in the resonator and the transmission of the fundamental-mode signal over a wide parameter range exploiting resonances with the dressed qubit. Experiments show that in the case of high quality resonators the dressed energy levels and corresponding resonance conditions can be probed, even for high driving amplitudes. The interaction of the qubit with photons of two harmonics can be used for the creation of quantum amplifiers or attenuators.
\end{abstract}

\section{INTRODUCTION}

A number of experiments with strongly driven superconducting qubits have been interpreted in terms of interference between multiple Landau-Zener trajectories $\stackrel{1}{\underline{1}}$ or multiphoton transitions ${ }^{2}$. Several years ago, Chalmers group demonstrated that the dynamics of a qubit in an intense microwave field can be convincingly described by the dressed-state model ${ }^{3}$. Indeed, a system composed of qubits coupled to the electromagnetic fields in resonators, represents a mesoscopic analogue of atoms coupled to light fields in optical cavities, and similar effects have been studied on atomic systems $4-6$. Due to this analogy the mathematical apparatus applied for describing the atomic systems, can be adapted to the mesoscopic ones.

In this paper, we consider the situation of a qubit coupled to two driving fields. Similar doubly-driven systems have already been used for the two-tone spectroscopy ${ }^{7}$ (also referred to as the pump-probe technique $\left.{ }^{8,9}\right)$. Here, by driving the qubit with one and probing the system at another (probe) frequency the energy level structure can be reconstructed. It can be realized experimentally by making use of two excited resonators ${ }^{10,11}$ or two modes of the same resonator [3, 12-14].

The notion of dressed states ${ }^{15-18}$ can be extended to two driving signals. In this case, one can describe the aggregate system in terms of the doubly-dressed states. ${ }^{19-21}$ Note, that this approach is in contrast to the Floquet method, in which the Floquet states make the quasi-classical counterpart of the dressed states and their energies $9,22,23$.

In particular, due to the energy and information transfer between the subsystems, the designated signal can be amplified or attenuated. ${ }^{15}$ Indeed, recently the amplification and attenuation of a probe signal were studied in a number of experiments [24-26]. Such processes can be important for microwave engineering, including quantum amplifiers and atten-

\footnotetext{
* These authors contributed equally to this work
}

uators. In the work by Oelsner et al. ${ }^{25}$ such kind of amplification was demonstrated. Here, the dynamics is as follows: one mode induces Rabi oscillations in the qubit and, when the frequency of these oscillations is adjusted to the second signal frequency, resonant interaction can result in amplification or damping of the latter signal. $27-30$ For a practical realization, the Rabi frequency is tuned into resonance with the oscillator and thus provides a qubit-resonator energy exchange. Similar effects were detected a long time ago ${ }^{31}$ as NMR-amplification at the Rabi frequency. Since the solid state quantum systems are scalable and tunable, the effect of direct Rabi transitions can be used for microwave quantum engineering.

The purpose of this article is to study in detail the doublydriven qubit - resonator system and to demonstrate its application to a realistic system. To this end, we develop a theoretical formalism to describe the transmission of a probe signal at the resonator's fundamental frequency. Our approach is general and valid over a wide range of parameters. In particular, the weak-probe limit can be relevant for probing the energy levels of the qubit-resonator system. We not only discuss this case close to the Rabi frequency, but also consider the resonant excitation of the coalesced system for both strong driving and strong probing signals. This allows us to derive analytic results for the qubit dressed, firstly, by the driving field and, secondly, by the probing field. Importantly, our formalism explains qualitatively and quantitatively the experimental results.

The rest of the paper is organized as follows. In Sec. II the Hamiltonian of the qubit interacting with two modes of the driven resonator is introduced and the energy spectrum of the total system is calculated. The details of those derivations are presented in the Appendix, where it is shown that the aggregate system after transformations can be described as a dressed qubit. The same transformations modify the master equation, which includes the relaxation rates, as it is described in Sec. III. The solution of the Lindblad equation with the parameter-dependent relaxation rates is the subject of Sec. IV. In particular, it is demonstrated that the driving in 
one harmonic influences the signal at other harmonics via the qubit. The corresponding experimental results are presented in Sec. V.

\section{DOUBLY-DRESSED STATES}

Consider a transmission-line $\lambda / 2$-resonator with a single flux qubit in it. The resonator is assumed to be driven by two signals: a low-amplitude probing signal, with a frequency $\omega_{\mathrm{p}}$ close to the resonator's fundamental frequency $\omega_{\mathrm{r}}$, and a high-amplitude driving signal, with a frequency $\omega_{\mathrm{d}}$. The driving frequency is considered to be close to the third harmonic frequency, and hence one has to take into account this very harmonic component in the Hamiltonian.

In the experimental realization ${ }^{25,32}$ the observables relate to the fundamental mode and the system can be described by the reduced Hamiltonian traced over the third-harmonic resonator mode. In the Appendix we have rewritten the total system's Hamiltonian so that it includes the strong driving signal as the renormalization of the qubit's Hamiltonian, which can be interpreted in terms of the dressed states. There, we start from the bare qubit, characterized by $H_{\mathrm{qb}}=-\frac{\Delta}{2} \tau_{x}-\frac{\varepsilon_{0}}{2} \tau_{z}$ in terms of the Pauli matrices $\tau_{i}$; it has the bare energy level distance $\Delta E=\sqrt{\Delta^{2}+\varepsilon_{0}^{2}}$. The qubit is considered to be coupled to the two-mode resonator and the coupling characterized by the value $\mathrm{g}_{1}$. So, the total Hamiltonian in the rotating-wave approximation (RWA) is written as follows (see Eqs. A9, A14, A18):

$$
\begin{aligned}
\widetilde{H}= & -\frac{\widetilde{\varepsilon}}{2} \sigma_{z}+\frac{\widetilde{\Delta}}{2} \sigma_{x}+\hbar \omega_{\mathrm{r}} a^{\dagger} a-\hbar \mathrm{g}_{\varepsilon}\left(a+a^{\dagger}\right) \sigma_{z}+ \\
& +\xi_{\mathrm{p}}\left(a e^{i \omega_{\mathrm{p}} t}+a^{\dagger} e^{-i \omega_{\mathrm{p}} t}\right)
\end{aligned}
$$

This describes the dressed qubit interacting with the resonator with the renormalized coupling $\mathrm{g}_{\varepsilon}=\mathrm{g}_{1} \varepsilon_{0} / \Delta E$ and probed by the signal with the amplitude $\xi_{\mathrm{p}}$. The qubit is now described by the Pauli matrices $\sigma_{i}$ and the resonator is described by the photon annihilation and creation operators, $a$ and $a^{\dagger}$. The dressed bias $\widetilde{\varepsilon}$ and the tunneling amplitude $\widetilde{\Delta}$ are defined by the driving frequency $\omega_{\mathrm{d}}$ and amplitude $A_{\mathrm{d}}$ either in the weak-driving regime, at $A_{\mathrm{d}}<\hbar \omega_{\mathrm{d}}$,

$$
\widetilde{\varepsilon}=\Delta E-\hbar \omega_{\mathrm{d}}, \widetilde{\Delta}=\Delta A_{\mathrm{d}} / 2 \Delta E,
$$

or in the strong-driving regime, where the energy bias is defined by the detuning from the $k$-photon resonance, $\widetilde{\varepsilon} \rightarrow \widetilde{\varepsilon}^{(k)}$, and the renormalized tunneling amplitude is defined by the oscillating Bessel function, $\widetilde{\Delta} \rightarrow \widetilde{\Delta}^{(k)}$, as follows

$$
\widetilde{\varepsilon}^{(k)}=\Delta E-k \hbar \omega_{\mathrm{d}}, \widetilde{\Delta}^{(k)}=\Delta \frac{k \hbar \omega_{\mathrm{d}}}{\varepsilon_{0}} J_{k}\left(\frac{A_{\mathrm{d}}}{\hbar \omega_{\mathrm{d}}} \frac{\varepsilon_{0}}{\Delta E}\right) .
$$

These values define the dressed energy levels, $\widetilde{E}_{ \pm}= \pm \widetilde{\Delta E} / 2$, where the distance between the energy levels is

$$
\widetilde{\Delta E}=\sqrt{\widetilde{\varepsilon}^{2}+\widetilde{\Delta}^{2}}
$$

which gives the dressed Rabi frequency $\Omega_{\mathrm{R}}=\widetilde{\Delta E} / \hbar$. The Hamiltonian (1) directly brings us to the problem of a qubit interacting with a weakly-driven fundamental-mode resonator, e.g. [33], with the following changes of notation for the bias and gap in the Hamiltonian with the dressed ones: $\varepsilon_{0} \rightarrow \widetilde{\varepsilon}$ and $\Delta \rightarrow \widetilde{\Delta}$.

Diagonalization of the time-independent part of the Hamiltonian (11), i.e. at $\xi_{\mathrm{p}}=0$, gives the energy levels for the system of the qubit coupled to two modes of the resonator - the doubly-dressed states:

$$
\begin{aligned}
E_{ \pm, n} & =\hbar \omega_{\mathrm{r}}(n+1) \pm \frac{\hbar \Omega_{n}}{2}, E_{\mathrm{gr}}=-\frac{\hbar \delta \widetilde{\omega}_{\mathrm{qb}}}{2} \\
\Omega_{n} & =\sqrt{4 \widetilde{\mathrm{g}}^{2}(n+1)+\delta \widetilde{\omega}_{\mathrm{qb}}^{2}}, \quad n=0,1,2, \ldots, \\
\delta \widetilde{\omega}_{\mathrm{qb}} & =\widetilde{\Delta E} / \hbar-\omega_{\mathrm{p}}, \widetilde{\mathrm{g}}=\mathrm{g}_{1} \frac{\varepsilon_{0}}{\Delta E} \frac{\widetilde{\Delta}}{\widetilde{\Delta E}} .
\end{aligned}
$$

The probing signal is described by the last term in Hamiltonian (1). When the photon energy $\hbar \omega_{\mathrm{p}}$ equals the energy difference $E_{ \pm, n}-E_{\mathrm{gr}}$, resonant energy exchange between the probing signal and the doubly-dressed states takes place. This resonant condition then reads

$$
\omega_{\mathrm{r}} n \pm \sqrt{\widetilde{\mathrm{g}}^{2}(n+1)+\left(\frac{\delta \widetilde{\omega}_{\mathrm{qb}}}{2}\right)^{2}}+\frac{\delta \widetilde{\omega}_{\mathrm{qb}}}{2}=\omega_{\mathrm{p}}-\omega_{\mathrm{r}} .
$$

Whether the energy exchange bear an amplifying or attenuating character depends on the relaxation parameters, which is the subject of the next section.

In particular, at weak resonant probe $\left(\omega_{\mathrm{p}}=\omega_{\mathrm{r}}\right)$ and when the coupling is negligible $\left(\mathrm{g}_{1} \ll \omega_{\mathrm{r}}, \Delta\right)$, from Eq. (8) we obtain two sorts of resonance conditions (in agreement with the results of the semi-classical Floquet formalism 9.34). The first one does not include resonator excitation: $n=0$ and

$$
\delta \widetilde{\omega}_{\mathrm{qb}}=0 .
$$

This corresponds to direct exchange of excitation between the probing signal and the dressed qubit: $\hbar \omega_{\mathrm{p}}=\widetilde{\Delta E}$. In other words, the probing signal frequency matches the qubit's Rabi frequency: $\omega_{\mathrm{p}}=\Omega_{\mathrm{R}}=\widetilde{\Delta E} / \hbar$. The second resonance condition involves the resonator excitation: $n=1$ and

$$
\delta \widetilde{\omega}_{\mathrm{qb}}=\omega_{\mathrm{p}} .
$$

This describes the two-photon process at $2 \hbar \omega_{\mathrm{p}}=\widetilde{\Delta E} .30$

We note that the resonant conditions (9-10) can alternatively be obtained by neglecting in Eq. (1) the qubit-resonator interaction and the probing signal, $\mathrm{g}=\xi_{\mathrm{p}}=0$. Then we obtain the energy levels for the qubit-resonator system $E_{ \pm, n}^{0}=$ $\pm \widetilde{\Delta E} / 2+\hbar \omega_{\mathrm{r}} n$. The resonant condition which involves the energy exchange between the probing signal and the dressed qubit, $\hbar \omega_{\mathrm{p}}=E_{+, n}^{0}-E_{-, n^{\prime}}^{0}$, gives at weak resonant probing $\left(\omega_{\mathrm{p}}=\omega_{\mathrm{r}}\right)$ two sorts of resonances. The first one does not include resonator excitation: $n=n^{\prime}$. This corresponds to Eq. (9). In other words, the probing signal frequency matches the qubit's Rabi frequency: $\omega_{\mathrm{p}}=\Omega_{\mathrm{R}}=\widetilde{\Delta E} / \hbar$. The second 

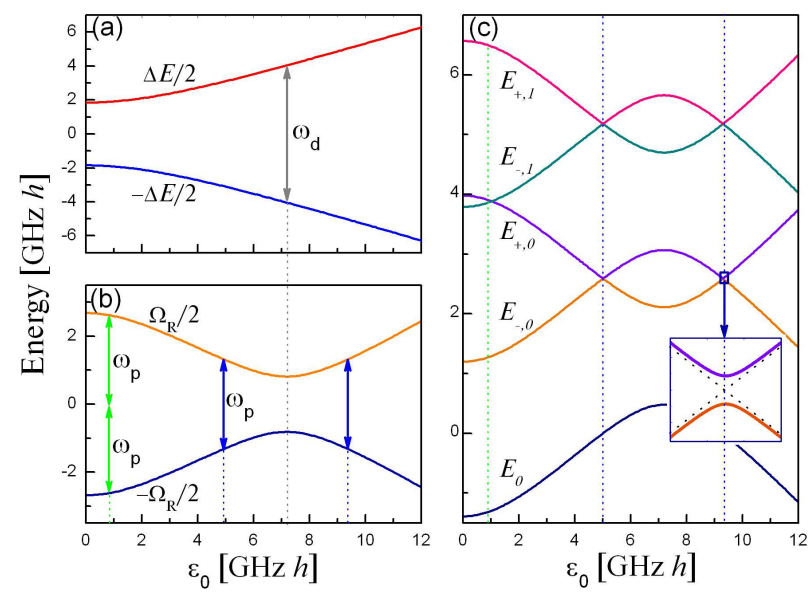

FIG. 1: (Color online) Bare, dressed, and doubly-dressed energy levels. The bare qubit's energy levels, $\pm \Delta E / 2$, are shown in (a); when they are matched by the driving frequency $\omega_{\mathrm{d}}$, the qubit is resonantly excited. (At higher values of the bias $\varepsilon_{0}$, the bare-qubit multiphoton excitation should be studied.) The position of the resonance, $\hbar \omega_{\mathrm{d}}=\Delta E$, is described by the avoided crossing of the dressed-state levels; the dressed and averaged energy levels, $\pm \widetilde{\Delta E} / 2= \pm \hbar \Omega_{\mathrm{R}} / 2$, are plotted in panel (b). When the dressed energy levels are matched by the second (probe) signal, $\hbar \omega_{\mathrm{p}}=\widetilde{\Delta E}$, a resonance interaction of the coalesced system is expected, Eq. (9). Also a resonance condition is given by the two-photon process (10). This is visualized as the avoided crossings of the doubly-dressed states, plotted in panel (c).

sort of resonances involves the resonator excitation: $n \neq n^{\prime}$. For example, for $n-n^{\prime}=1$ we have $2 \hbar \omega_{\mathrm{p}}=\widetilde{\Delta E}$, which describes the two-photon process.

For the following calculations we switch to the dressedqubit eigenstates and after another RWA related to the probing signal we obtain the time-independent Hamiltonian in the form (as it is described in the Appendix).

$H_{\mathrm{RWA}}=\hbar \frac{\delta \widetilde{\omega}_{\mathrm{qb}}}{2} \widetilde{\sigma}_{z}+\hbar \delta \omega_{\mathrm{r}} a^{\dagger} a+\hbar \widetilde{\mathrm{g}}\left(a \widetilde{\sigma}^{\dagger}+a^{\dagger} \widetilde{\sigma}\right)+\xi_{\mathrm{p}}\left(a+a^{\dagger}\right)$,

where $\delta \omega_{\mathrm{r}}=\omega_{\mathrm{r}}-\omega_{\mathrm{p}}$ and $\widetilde{\sigma}=\frac{1}{2}\left(\widetilde{\sigma}_{x}-i \widetilde{\sigma}_{y}\right)$ is the qubit lowering operator and here tilde relates to the new basis.

Finally, in Fig. 11 we illustrate the energy levels studied in this section. The parameters for the graph were taken for the system of Ref. [25]: $\Delta / h=3.7 \mathrm{GHz}, \omega_{\mathrm{r}} / 2 \pi=2.59 \mathrm{GHz}$ and $\omega_{\mathrm{d}} / 2 \pi=3 \omega_{\mathrm{r}} / 2 \pi=7.77 \mathrm{GHz}, A_{\mathrm{d}} / h=7 \mathrm{GHz}, \mathrm{g}_{1} / 2 \pi=0.8$ $\mathrm{MHz}$, and $\omega_{\mathrm{p}}=\omega_{\mathrm{r}}$. Figure 1 can be seen as the graphical description of dressing the dressed qubit, which can be considered as the mesoscopic tunable analogue of the atomic systems, as in Ref. [35]. We also note that for the small value of the coupling taken $\left(g_{1} \ll \omega_{\mathrm{r}}, \Delta\right)$, the splitting (which defines the difference between the dressed and doubly-dressed levels) is small, as shown in the inset. The solid lines describe the doubly-dressed levels, while the dotted lines are for the dressed levels, $\pm \widetilde{\Delta E} / 2+n \hbar \omega_{\mathrm{r}}$.

\section{III. "DRESSED RELAXATION"}

The Lindblad equation for the system can be written in terms of the bare qubit and resonator states as follows [36]

$$
\dot{\rho}=-\frac{i}{\hbar}[H, \rho]+\sum_{\alpha} \mathcal{L}_{\alpha}[\rho],
$$

where the index $\alpha$ numerates different relaxation channels and the respective Lindbladian superoperators are given by the formulas

$$
\begin{aligned}
& \mathcal{L}_{\kappa}=\kappa\left(a \rho a^{\dagger}-\frac{1}{2}\left\{a^{\dagger} a, \rho\right\}\right), \\
& \mathcal{L}_{\downarrow}=\Gamma_{1}\left(\sigma \rho \sigma^{\dagger}-\frac{1}{2}\left\{\sigma^{\dagger} \sigma, \rho\right\}\right), \\
& \mathcal{L}_{\phi}=\frac{\Gamma_{\phi}}{2}\left(\sigma_{z} \rho \sigma_{z}-\rho\right) .
\end{aligned}
$$

These could also be written in the unified form

$$
\begin{aligned}
\mathcal{L}_{\alpha} & =L_{\alpha} \rho L_{\alpha}^{\dagger}-\frac{1}{2}\left\{L_{\alpha}^{\dagger} L_{\alpha}, \rho\right\}= \\
& =\frac{1}{2}\left[L_{\alpha} \rho, L_{\alpha}^{\dagger}\right]+\frac{1}{2}\left[L_{\alpha}, \rho L_{\alpha}^{\dagger}\right] .
\end{aligned}
$$

with the operators $L_{\alpha}$ given by the following: $L_{\kappa}=\sqrt{\kappa} a$, $L_{\downarrow}=\sqrt{\Gamma_{1}} \sigma, L_{\phi}=\sqrt{\Gamma_{\phi} / 2} \sigma_{z}$. Here $\kappa$ is the decay rate of the photons in the resonator, $\Gamma_{1}$ and $\Gamma_{\phi}$ are the qubit's relaxation and dephasing rates.

After the transformations described in the previous section we arrive at the Lindblad equation in the form of Eq. (12) with the dressed Hamiltonian $H_{\mathrm{RWA}}$ and with the dressed relaxation terms: $\widetilde{\mathcal{L}}=\mathcal{L}_{\kappa}+\widetilde{\mathcal{L}}_{\downarrow}+\widetilde{\mathcal{L}} \uparrow+\widetilde{\mathcal{L}}_{\phi}$,

$$
\begin{aligned}
\widetilde{\mathcal{L}}_{\downarrow} & =\widetilde{\Gamma_{\downarrow}}\left(\widetilde{\sigma} \widetilde{\rho \sigma} \widetilde{\sigma}^{\dagger}-\frac{1}{2}\left\{\widetilde{\sigma}^{\dagger} \widetilde{\sigma}, \widetilde{\rho}\right\}\right), \\
\widetilde{\mathcal{L}}_{\uparrow} & =\widetilde{\Gamma_{\uparrow}}\left(\widetilde{\sigma}^{\dagger} \widetilde{\rho} \widetilde{\sigma}-\frac{1}{2}\left\{\widetilde{\sigma} \widetilde{\sigma}^{\dagger}, \widetilde{\rho}\right\}\right), \\
\widetilde{\mathcal{L}}_{\phi} & =\frac{\widetilde{\Gamma_{\phi}}}{2}\left(\widetilde{\sigma}_{z} \widetilde{\rho} \widetilde{\sigma}_{z}-\widetilde{\rho}\right) .
\end{aligned}
$$

[Namely, to obtain these formulas, we applied the transformation $\widetilde{S}$ (see Appendix) to the original Lindbladians and kept only the terms which survive under the RWA; this means keeping only the terms, which contain $\widehat{1}, \widetilde{\sigma}_{z}$, or the product of $\widetilde{\sigma}$ and $\widetilde{\sigma}^{\dagger}$.] Thus, the dressed Lindbladian superoperators in the sum $\sum_{\alpha} \widetilde{\mathcal{L}}_{\alpha}[\widetilde{\rho}]$ can be written in the compact form of Eq. (16), where $L_{\kappa}=\sqrt{\kappa} a, L_{\downarrow}=\sqrt{\widetilde{\Gamma_{\downarrow}}} \widetilde{\sigma}, L_{\uparrow}=\sqrt{\widetilde{\Gamma_{\uparrow}}} \widetilde{\sigma}^{\dagger}$, $L_{\phi}=\sqrt{\widetilde{\Gamma_{\phi}} / 2} \widetilde{\sigma}_{z}$. Here the tunable dressed rates for relaxation, excitation and dephasing ${ }^{30,37}$ are given by the expressions

$$
\begin{aligned}
\widetilde{\Gamma}_{\downarrow / \uparrow} & =\frac{\Gamma_{1}}{4}\left(1 \pm \frac{\widetilde{\varepsilon}}{\widetilde{\Delta E}}\right)^{2}+\frac{\Gamma_{\phi}}{2} \frac{\widetilde{\Delta}^{2}}{\widetilde{\Delta E}^{2}} \\
\widetilde{\Gamma}_{\phi} & =\frac{\Gamma_{1}}{2} \frac{\widetilde{\Delta}^{2}}{\widetilde{\Delta E}^{2}}+\Gamma_{\phi} \frac{\widetilde{\varepsilon}^{2}}{\widetilde{\Delta E}^{2}} .
\end{aligned}
$$


The Lindbladian $\mathcal{L}_{\kappa}$ describes the relaxation in the resonator, $\widetilde{\mathcal{L}}_{\downarrow / \uparrow}$ describe both, the relaxation and excitation in the qubit, while $\widetilde{\mathcal{L}}_{\phi}$ relates to the dephasing. ${ }^{30}$ Note that these "dressed" relaxation rates appear due to taking into account photons of the driving field, but neglecting the weak probing signal.

From Eq. 20, one can estimate the interplay of the relaxation and excitation in the driven qubit. We note that the difference between the excitation and relaxation rates is

$$
\widetilde{\Gamma}_{\uparrow}-\widetilde{\Gamma}_{\downarrow}=\Gamma_{1} \widetilde{\varepsilon} / \widetilde{\Delta E}
$$

and the rates are equal at $\widetilde{\varepsilon}=0$, while for the red and blue detuning, at $\widetilde{\varepsilon} \gtrless 0$ we have $\widetilde{\Gamma}_{\uparrow} \gtrless \widetilde{\Gamma}_{\downarrow}$ and either excitation or relaxation dominates.

In the limit of strong driving, the qubit's Hamiltonian is given by Eq. (A22), which coincides in form with Eq. A18). This allows us immediately to obtain the dressed relaxation rates in the vicinity of the $k$-th resonance (where $\Delta E-$ $\left.k \hbar \omega_{\mathrm{d}} \ll \Delta E\right)$ by simply replacing $\widetilde{\varepsilon} \rightarrow \widetilde{\varepsilon}^{(k)}$ and $\widetilde{\Delta} \rightarrow \widetilde{\Delta}^{(k)}$ in the formulas 20,21).

To understand the impact of the effective relaxation terms, we consider the reduction of the Lindblad equation to the Bloch-type one, similar as in Ref. [38]. Therefore, we start with the Lindblad equation for the density matrix of the dressed qubit (neglecting the resonator fundamental mode): $\widetilde{\rho}=\sum_{i, j=0,1} \rho_{i j}|i\rangle\langle j|$. Then from Eqs. (12] 17, 19] we obtain for the free qubit evolution (taking into account that $\left.\rho_{00}=1-\rho_{11}\right)$ :

$$
\begin{aligned}
& \dot{\rho}_{11}=-\left(\widetilde{\Gamma}_{\uparrow}+\widetilde{\Gamma}_{\downarrow}\right) \rho_{11}+\widetilde{\Gamma}_{\uparrow}, \\
& \dot{\rho}_{01}=-\widetilde{\Gamma}_{2} \rho_{01}, \quad \widetilde{\Gamma}_{2}=\widetilde{\Gamma}_{\phi}+\frac{\widetilde{\Gamma}_{\uparrow}+\widetilde{\Gamma}_{\downarrow}}{2} .
\end{aligned}
$$

From here, in particular, it follows that the equilibrium population of the excited state is defined by the rate $\widetilde{\Gamma}_{\uparrow}: \rho_{11}^{e q .}=$ $\widetilde{\Gamma}_{\uparrow} /\left(\widetilde{\Gamma}_{\uparrow}+\widetilde{\Gamma}_{\downarrow}\right)$.

\section{IMPACT OF THE QUBIT'S DRIVING ON THE TRANSMITTED SIGNAL}

The dynamical behavior of the system is described by the Lindblad equation (12) in combination with the Hamiltonian (11). The stationary solution can be found by assuming $\dot{\rho}=0$. In the limit of small driving amplitude, as e.g. in Ref. [33], an analytic solution is possible.

From Eq. (12) we obtain the equation of motion for the expectation value of any quantum operator $A$

$$
\frac{d\langle A\rangle}{d t}=-\frac{i}{\hbar}\left\langle\left[A, H_{\mathrm{RWA}}\right]\right\rangle+\operatorname{Tr}(A \widetilde{\mathcal{L}})
$$

where $\langle A\rangle=\operatorname{Tr}(A \rho),\langle[A, H]\rangle=\operatorname{Tr}([A, H] \rho)$, the trace is over all eigenstates of the system. In our system, the trace in Eq. (24) is over the photon states of the fundamental mode $|n\rangle$ and the two qubit states $| \pm\rangle$. For the expectation values of the operators $a, \widetilde{\sigma}, \widetilde{\sigma}_{z}, n=a^{\dagger} a$ we obtain the following system of equations (also called Maxwell-Bloch equations):

$$
\begin{aligned}
\frac{d\langle a\rangle}{d t} & =-i \delta \omega_{\mathrm{r}}^{\prime}\langle a\rangle-i \widetilde{\mathrm{g}}\langle\widetilde{\sigma}\rangle-i \frac{\xi_{\mathrm{p}}}{\hbar} \\
\frac{d\langle\widetilde{\sigma}\rangle}{d t} & =-i \delta \widetilde{\omega}_{\mathrm{qb}}^{\prime}\langle\widetilde{\sigma}\rangle+i \widetilde{\mathrm{g}}\left\langle a \widetilde{\sigma}_{z}\right\rangle \\
\frac{d\left\langle\widetilde{\sigma}_{z}\right\rangle}{d t} & =-i 2 \widetilde{\mathrm{g}}\left(\left\langle a \widetilde{\sigma}^{\dagger}\right\rangle-\left\langle a^{\dagger} \widetilde{\sigma}\right\rangle\right)-\widetilde{\Gamma}_{+}\left\langle\widetilde{\sigma}_{z}\right\rangle-\widetilde{\Gamma}_{-}
\end{aligned}
$$

$$
\frac{d\left\langle a \widetilde{\sigma}^{\dagger}\right\rangle}{d t}=i(\delta \omega+i G)\left\langle a \widetilde{\sigma}^{\dagger}\right\rangle-i \widetilde{\mathrm{g}}\left(\left\langle\widetilde{\sigma}^{\dagger} \widetilde{\sigma}\right\rangle+\left\langle a^{\dagger} a \widetilde{\sigma}_{z}\right\rangle\right)-i \frac{\xi_{\mathrm{p}}}{\hbar} \widetilde{\sigma}^{\dagger},
$$

$$
\begin{aligned}
\frac{d\left\langle a \widetilde{\sigma}_{z}\right\rangle}{d t}= & -\left(i \delta \omega_{\mathrm{r}}+Q\right)\left\langle a \widetilde{\sigma}_{z}\right\rangle+i \widetilde{\mathrm{g}}\langle\tilde{\sigma}\rangle+\widetilde{\Gamma}_{-}\langle a\rangle \\
& -i \frac{\xi_{\mathrm{p}}}{\hbar}\left\langle\widetilde{\sigma}_{z}\right\rangle-2 i \widetilde{\mathrm{g}}\left\langle a a \tilde{\sigma}^{\dagger}\right\rangle+2 i \widetilde{\mathrm{g}}\left\langle a^{\dagger} a \tilde{\sigma}\right\rangle
\end{aligned}
$$

$$
\frac{d\langle n\rangle}{d t}=-\kappa\langle n\rangle+i \widetilde{\mathrm{g}}\left(\left\langle a \tilde{\sigma}^{\dagger}\right\rangle-\left\langle a^{\dagger} \widetilde{\sigma}\right\rangle\right)+\frac{i}{\hbar} \xi_{\mathrm{p}}\left(\langle a\rangle-\left\langle a^{\dagger}\right\rangle\right),
$$

where $\widetilde{\Gamma}_{ \pm}=\widetilde{\Gamma}_{\downarrow} \pm \widetilde{\Gamma}_{\uparrow}, \delta \omega_{\mathrm{r}}^{\prime}=\delta \omega_{\mathrm{r}}-i \kappa / 2, \delta \widetilde{\omega}_{\mathrm{qb}}^{\prime}=\delta \widetilde{\omega}_{\mathrm{qb}}-i \widetilde{\Gamma}_{2}$, $\delta \omega=\omega_{\mathrm{qb}}-\omega_{\mathrm{r}}, G=\widetilde{\Gamma}_{2}+\kappa / 2, Q=\kappa / 2+\widetilde{\Gamma}_{+}$. These equations present only the first few of the infinite ladder of coupled equations, which include the higher-order correlations.

The stationary solution for this system can be found analytically if we restrict the Hilbert space by $n=0,1$ states only, which is justified for small amplitude probe signals (see, for example, Ref. [33]). However, in real experiments the number of fundamental photons is not so low ${ }^{45}$. In order to study this case we analyze below the full set of equations 25,30$)$.

By solving these equations we can obtain the transmission, as probed by a network analyzer, which is defined in the way adopted in quantum optics $12,26,33$

$$
t=i \frac{\hbar \kappa}{2 \xi_{\mathrm{p}}}\langle a\rangle
$$

First we find the stationary solutions to the above equations for the case when the interaction between the qubit and the resonator is absent, $\widetilde{\mathrm{g}}=0$. We obtain $\langle\widetilde{\sigma}\rangle_{0}=0$,

$$
\begin{gathered}
\langle a\rangle_{0}=-\frac{\xi_{\mathrm{p}}}{\hbar \delta \omega_{r}^{\prime}}, \\
\left\langle\widetilde{\sigma}_{z}\right\rangle_{0}=-\frac{\widetilde{\Gamma}_{-}}{\widetilde{\Gamma}_{+}} .
\end{gathered}
$$

From Eq. 32 we obtain the transmission amplitude for the bare resonator

$$
\left|t_{0}\right|=\frac{\hbar \kappa}{2 \xi_{\mathrm{p}}}\left|\langle a\rangle_{0}\right|=\frac{\kappa}{\sqrt{\kappa^{2}+4 \delta \omega_{\mathrm{r}}^{2}}},
$$

and from Eq. (30) we obtain the average photon number

$$
\langle n\rangle_{0}=-\frac{\xi_{\mathrm{p}}}{\hbar \kappa} 2 \operatorname{Im}\langle a\rangle_{0}=\frac{4 \xi_{\mathrm{p}}^{2}}{\hbar^{2}\left(4 \delta \omega_{\mathrm{r}}^{2}+\kappa^{2}\right)} .
$$


It follows that the condition for small $\langle n\rangle$ is $\xi_{\mathrm{p}}^{2} / \hbar^{2} \kappa^{2} \ll 1$.

In order to proceed further, we truncate the above infinite system of equations. In the equations (28) and (29) we neglect the correlations which contain three operators $\left\langle a^{\dagger} a \widetilde{\sigma}_{z}\right\rangle$, $\left\langle a a \widetilde{\sigma}^{\dagger}\right\rangle,\left\langle a^{\dagger} a \widetilde{\sigma}\right\rangle$. This is justified if $\langle n\rangle$ is small. In terms of the density matrix this condition is equivalent to $\langle 0|\rho| 0\rangle \gg$ $\langle n|\rho| n\rangle$, where $n \neq 0$. This means that the state $n=0$ is more populated than other states with $n \neq 0$. For example, in Eq. (28) the term $\left\langle a^{\dagger} a \widetilde{\sigma}_{z}\right\rangle$ is neglected as compared with $\left\langle\widetilde{\sigma}^{\dagger} \widetilde{\sigma}\right\rangle=\left(1+\left\langle\widetilde{\sigma}_{z}\right\rangle\right) / 2$.

Next, we consider the qubit-resonator system in the absence of an external probe, $\xi_{\mathrm{p}}=0, \widetilde{\mathrm{g}} \neq 0$. Then from the steadystate solution of Eqs. (27) and (28) we find the steady-state polarization of the qubit in the resonator:

$$
\left\langle\widetilde{\sigma}_{z}\right\rangle_{0}=-\frac{\widetilde{\Gamma}_{-}+A}{\widetilde{\Gamma}_{+}+A},
$$

where

$$
A=\frac{2 \widetilde{\mathrm{g}}^{2} G}{(\delta \omega)^{2}+G^{2}},
$$

and the average photon number in fundamental mode:

$$
\langle n\rangle_{\xi_{\mathrm{p}}=0}=\frac{1}{\kappa} \frac{A \widetilde{\Gamma}_{1}}{\widetilde{\Gamma}_{+}+A} .
$$

Finally, we analyze the full set of truncated equations 25 30). Considering the probing signal $\xi_{\mathrm{p}}$ to be weak, the qubit operators acquire only small corrections: $\left\langle\widetilde{\sigma}_{z}\right\rangle=\left\langle\widetilde{\sigma}_{z}\right\rangle_{0}+$ $O\left(\xi_{\mathrm{p}}^{2}\right),\langle\widetilde{\sigma}\rangle=O\left(\xi_{\mathrm{p}}\right)$. Hence, keeping the first-order approximation in $\xi_{\mathrm{p}}$ we obtain from equations (25), (26) and (29) the stationary solutions for the intracavity field

$$
\langle a\rangle=-\frac{\xi_{\mathrm{p}}}{\hbar} \frac{\delta \widetilde{\omega}_{\mathrm{qb}}^{\prime}}{S \widetilde{\mathrm{g}}^{2}+\delta \widetilde{\omega}_{\mathrm{qb}}^{\prime} \delta \omega_{\mathrm{r}}^{\prime}}
$$

where

$$
S=\frac{\delta \widetilde{\omega}_{\mathrm{qb}}^{\prime}\left[\widetilde{\Gamma}_{-}+i\left\langle\widetilde{\sigma}_{z}\right\rangle_{0} \delta \omega_{\mathrm{r}}^{\prime}\right]}{\widetilde{\mathrm{g}}^{2}\left(1+\left\langle\widetilde{\sigma}_{z}\right\rangle_{0}\right)+i \delta \widetilde{\omega}_{\mathrm{qb}}^{\prime}\left(\delta \omega_{\mathrm{r}}^{\prime}-i \widetilde{\Gamma}_{+}\right)} .
$$

Equation (39) together with Eq. (31) defines the transmission amplitude. In particular, when the excitation rate is disregarded, $\widetilde{\Gamma}_{\uparrow}=0$, we have $\left\langle\widetilde{\sigma}_{z}\right\rangle_{0}=-1$. If, in addition, the relaxation rates are small $\left(\widetilde{\Gamma}_{-}, \widetilde{\Gamma}_{+} \ll \delta \omega_{\mathrm{r}}^{\prime}\right)$ we get $S=-1$ and recover from (39) the result of Ref. [33] obtained in the singleexcitation regime (in the first approximation in the probingsignal amplitude $\xi_{\mathrm{p}}$ ).

From Eq. (30) the average number of the fundamentalmode photons can be found by:

$$
\begin{aligned}
\langle n\rangle & =-\frac{\xi_{\mathrm{p}}}{\hbar \kappa} 2 \operatorname{Im}\langle a\rangle+\frac{i \widetilde{\mathrm{g}}}{\kappa}\left(\left\langle a \widetilde{\sigma}^{\dagger}\right\rangle-\left\langle a^{\dagger} \widetilde{\sigma}\right\rangle\right) \\
& =\frac{\xi_{\mathrm{p}}^{2}}{\hbar^{2} \kappa} \frac{\kappa\left|\delta \omega_{\mathrm{qb}}\right|^{2}+i \widetilde{\mathrm{g}}^{2}\left(S \delta \omega_{\mathrm{qb}}^{\prime *}-S^{*} \delta \omega_{\mathrm{qb}}^{\prime}\right)}{\left|S \widetilde{\mathrm{g}}^{2}+\delta \omega_{\mathrm{qb}}^{\prime} \delta \omega_{\mathrm{r}}^{\prime}\right|^{2}}+\langle n\rangle_{\xi_{\mathrm{p}}=0} .
\end{aligned}
$$

where $\Gamma_{2}=\Gamma_{\phi}+\Gamma_{1} / 2$.

\section{EXPERIMENT}

To prove the theoretical description above, we fabricated a coplanar waveguide resonator with resonance frequency of $\omega_{\mathrm{r}} / 2 \pi=2.59 \mathrm{GHz}$ and a lossrate of $\kappa / 2 \pi=44 \mathrm{kHz}$. Two qubit loops, with sizes of $14 \times 7 \mu \mathrm{m}^{2}$ for the first and $5 \times 5 \mu$ $\mathrm{m}^{2}$ for the second qubit are placed in the center of the resonator. With a high quality factor of the resonator $(Q \approx$ 70000 ) and rather weak resonator-qubit couplings we ensure, that the decoherence of the coupled system is not significantly increased in comparison to that of a bare qubit. Indeed, we estimated the added width by the strong driving signal as $\sqrt{\langle N\rangle} \kappa$. So, even for photon numbers ranging up to one million it is still in the same order as the qubits decoherence, which is expected to be of the order of several $10 \mathrm{MHz}$. In that way the definition of true energy states and corresponding resonance conditions is justified. The sample was measured in a dilution refrigerator at a base temperature of about $20 \mathrm{mK}$. With independent measurements the minimal level spacings $\Delta^{(1)} / h=6 \mathrm{GHz}$ and $\Delta^{(2)} / h=9.4 \mathrm{GHz}$, the persistent currents $I_{\mathrm{p}}^{(1)}=60 \mathrm{nA}$ and $I_{\mathrm{p}}^{(2)}=100 \mathrm{nA}$, and the coupling constants $\mathrm{g}_{1}^{(1)} / 2 \pi=8 \mathrm{MHz}$ and $\mathrm{g}_{1}^{(2)} / 2 \pi=4 \mathrm{MHz}$ are defined for both qubits (similar to Ref. [32]). Here superscripts (1) and (2) denote the number of the qubit.

In a first set of experiments the bigger qubit was studied. A driving signal at the fifth harmonic of the resonator $\omega_{\mathrm{d}}=5 \omega_{\mathrm{r}}$ 


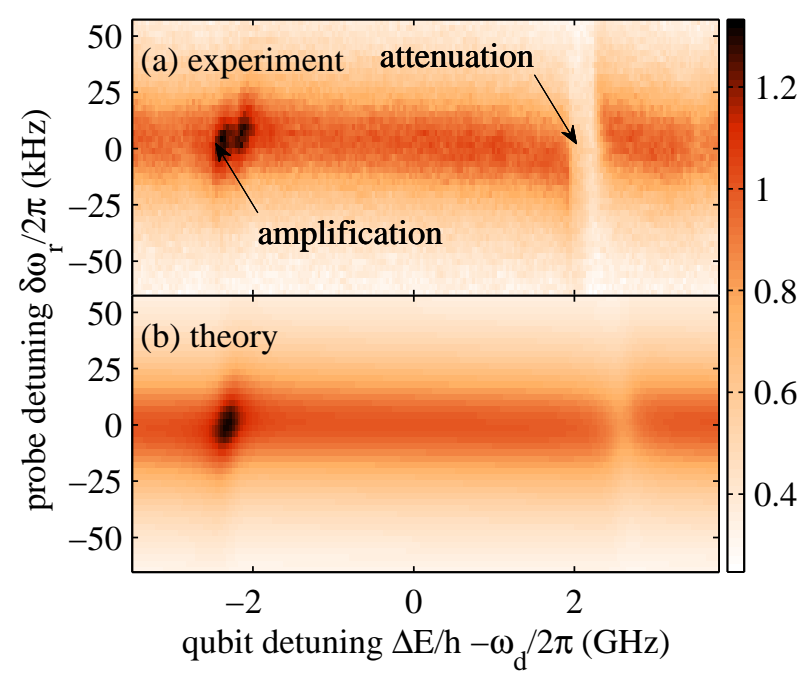

FIG. 2: (Color online) (a) Measured normalized transmission amplitude $|t|$ for the first qubit (see text) while a strong driving signal is applied. The results are plotted in dependence on the detuning between qubit frequency and driving signal, (controlled by the qubit bias $\varepsilon_{0}$ ), and the probing frequency detuning $\delta \omega_{\mathrm{r}}=\omega_{\mathrm{p}}-\omega_{\mathrm{r}}$. The amplification and the attenuation of the transmitted signal is found in agreement with the resonance conditions, $\hbar \omega_{\mathrm{p}}=\widetilde{\Delta E}$, Eq. (9). (b) Normalized transmission calculated for the same parameters as in (a) following Eq. 31) together with Eq. 39.

with a constant power was chosen. (We note that considering the fifth harmonic instead of the third does not change

In order to test our model in the strong driving regime, where Eq. (2) is replaced by Eq.(3), we analyze the response of the system as a function of the driving amplitude. Here we consider the case where the qubit gap is higher than the driving frequency. For this purpose the smaller qubit was used. The transmission at the fundamental mode, $\omega_{\mathrm{p}}=\omega_{\mathrm{r}}$, was measured while changing the frequency of the driving signal around the third harmonic frequency, and consequently the driving amplitude at the qubit. The results are shown in Fig. 3, together with calculated data following from Eq. (31). Several sharp lines of amplification (dark) and damping (light) were experimentally observed. The number of lines increases with increasing power and each of the lines corresponds to a resonance condition between the dressed states and the probing signal. To understand their origin, calculated transmission data was added into each plot. We split these theoretical plots into regions and for each of them use Eq. (31) with different index $k$ in Eq. (3). For high powers we note that the levels of one step of the dressed ladder are equivalent to the ones of the stairs above or below. In that way, we assume that Eq. (31) is valid also for those regions where resonances between the lower level of one stair and the higher level of the lower stair occur. To account for these interaction we replace the split- any point in the theoretical description of the system except of putting different values for the driving frequency.) The transmission was measured at small detunings $\delta \omega_{\text {r }}$ from the resonators fundamental mode, while the energy bias $\varepsilon_{0}$ of the qubit was varied. The experimental results are shown in Fig. 2 (a) together with simulations using Eq. 31 Fig. 2(b). It can be seen that a good agreement between the two was achieved for a relaxation rate $\Gamma_{1} / 2 \pi=4 \mathrm{MHz}$ and a pure dephasing $\Gamma_{\phi} / 2 \pi=200 \mathrm{MHz}$.

We find that if the qubit is detuned, the transmission amplitude is independent of the bias $\varepsilon_{0}$ and is described by the Lorentzian-shaped dependence on the frequency detuning, Eq. (34). When the frequency of the probing signal is close to the Rabi frequency $\omega_{\mathrm{p}} \approx \Omega_{\mathrm{R}}=\widetilde{\Delta E} / \hbar$, a resonant energy exchange between the qubit and the fundamental mode of the resonator results in amplification or attenuation of the transmitted signal at red or blue detuning $(\widetilde{\varepsilon} \gtrless 0)$ correspondingly. The observation of such amplification was recently reported by Oelsner et al. in Ref. [25]. This can be explained by the domination of relaxation or excitation, see Eqs. (20) and (22). Note that the inversion $\left\langle\widetilde{\sigma}_{z}\right\rangle$, which defines the difference between the occupation probabilities of the upper and lower qubit's levels, can be estimated with Eq. (33). This together with Eq. (22) gives positive and negative inversion for $\widetilde{\varepsilon}>0$ and $\widetilde{\varepsilon}<0$, respectively. The effect of amplification and attenuation of the transmitted signal can be related to the increase or decrease of the cavity photon number. In this sense one can term these as lasing and cooling of the resonator as in Ref. [30].

ting of the dressed states $\widetilde{\Delta E}^{(k)}$ by $\omega_{\mathrm{d}}-\widetilde{\Delta E}^{(k)}$. This makes it possible to relate each of the resonance lines to one index $k$ and to an interaction directly between the Rabi levels or levels of different stairs.

We find a quantitative agreement between the theoretical predictions and the experiment for a relaxation rate $\Gamma_{1}^{(2)} / 2 \pi=$ $6 \mathrm{MHz}$ and a pure dephasing $\Gamma_{\phi}^{(2)} / 2 \pi=100 \mathrm{MHz}$ of the second qubit. As examples we highlight several regions with black rectangles. Direct resonances between the Rabi levels are marked in plot (a) for $k=1$ and in (d) for $k=2$. Note, that for $k=1$ no amplification is observed since the driving frequency is chosen below the qubit gap. In Fig. 3 (d) the power dependence of the resonance lines for $k=2$ show a similar dependence as in Ref. [30]. The marked regions for damping in subplot ( $\mathrm{f}$ ) and amplification in $(\mathrm{g})$ correspond to interactions between levels of different stairs with the fundamental mode and indices $k=3$ and $k=2$, respectively.

The representation of Fig. 3 gives the possibility to follow the formation of the resonance lines and their change for increasing power. Note, that the slight differences in the positions, which become more pronounced for higher driving powers, are due to the limitation of our model: we only consider two dressed levels. Therefore, the dynamics of the sys- 


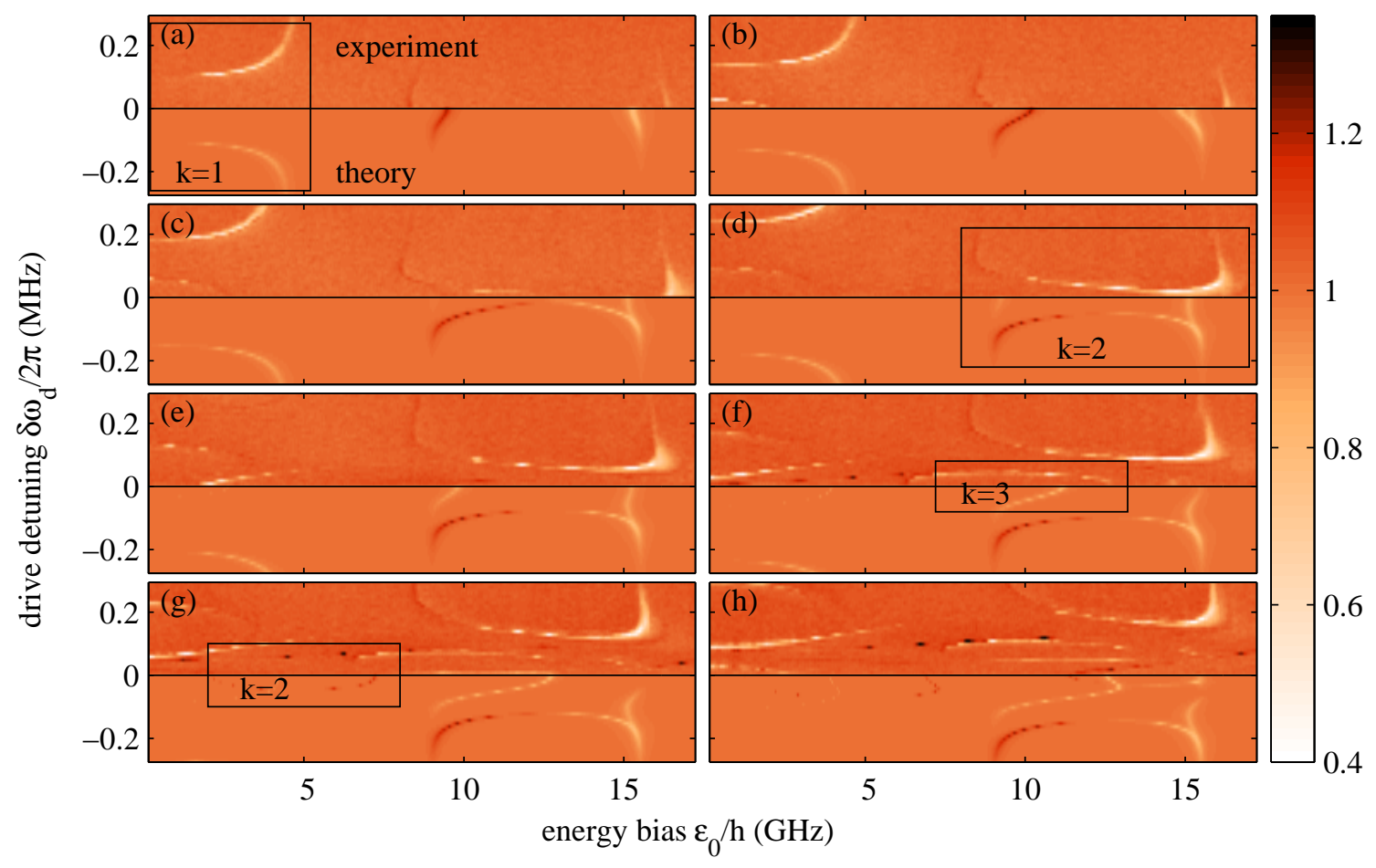

FIG. 3: (Color online) Normalized transmission amplitude through the resonator at a probing frequency $\omega_{\mathrm{p}}=\omega_{\mathrm{r}}$ for different driving amplitudes ranging from about $-131 \mathrm{dBm}$ in (a) to $-117 \mathrm{dBm}$ in $(\mathrm{g})$ in $2 \mathrm{dBm}$ steps at the input of the resonator. The transmission is plotted as a function of the energy bias $\varepsilon_{0}$ of the second qubit and the driving frequency detuning $\delta \omega_{\mathrm{d}}=\omega_{\mathrm{d}}-3 \omega_{\mathrm{r}}$. The driving frequency is changed only around the third harmonic in the order of its linewidth (about $6 \kappa$ ). The presented results correspond to a symmetric power dependence around the center frequency of the third harmonic, since the resonator acts as bandpass filter. Each plot is split into an experimental (positive detuning) and a theoretical (negative detuning) part. For the calculations the figures were split into regions for which Eq. (31) was used with a certain index $k$ in Eq. (3). Several features were highlighted with black rectangles to interrelate theory and experiment (see text).

tem contains only the influence of the $k$-photon resonance, and the theoretical predictions are close to the experiment where their influence dominates. For instance, when different resonance lines come close to each other, the two level approximation breaks down. For describing the full dynamics it would be necessary to either include more levels of the dressed ladder or to add corrections to the energy levels in one manifold (on one stair of the dressed ladder) introduced by the stairs above and below.

Our experimental results show, that in the strong driving limit levels of different photon numbers are strongly coupled and yield corrections to the level splitting of the Rabi levels. Furthermore, due to the strong mixing of the levels in the high driving case, resonance conditions can be defined between each adjacent pair of levels.

\section{CONCLUSIONS}

We have considered the situation when a two-level system (qubit) is coupled to two quantum oscillators. The emphasis was made on the specific solid-state realization of a flux qubit coupled to a transmission-line resonator, which is driven close to a harmonic and probed at the fundamental frequency. The former driving signal is not directly observable and was in- cluded into the considerations by the qubit's dressed states. Similarly, the interaction of the dressed qubit with the resonator's fundamental mode can be considered as the second dressing of the qubit's dressed states. When the energy of the probing photons matches the dressed energy levels, a resonant energy exchange results in either the amplification or attenuation of the probing signal, depending on the tunable relaxation rates. We have presented a detailed theoretical description of those processes, as well as related the results to other studies, such as Refs. [9, 30, 38]. Our theoretical findings together with the experimental results, presented here and in Ref. [25], are useful for the description of the qubit-resonator systems in terms of the dressed states. Especially since the high quality of the resonator together with the rather weak coupling between the resonator and the qubit prevent the energy levels from smearing out. In other words, although the driving signal is strong, the added decoherence is still small, so that sharp resonance conditions were observed. Furthermore, the idea of transferring energy from one resonator's mode to another via a single qubit may be useful for further applications. 


\section{Acknowledgments}

This work was partly supported by NAS of Ukraine (Project No. 4/13 -NANO), DKNII (Project No. M/231-2013), BMBF (UKR-2012-028).The research leading to these results has received funding from the European Community's Seventh Framework Programme (FP7/2007-2013) under grant agreement No. 270843. Ya.S.G. acknowledges the partial support from the Russian Ministry of Education and Science through the project TP 7.1667.2011 and from the German Ministry of Science (BMBF) through the project RUS 10/015. M.G. was supported by the Slovak Research and Development Agency under the contracts APVV-0515-10 and DO7RP-0032-11. SNS thanks S. Ashhab for useful comments. SNS and DSK acknowledge the hospitality of IPHT during their visits. We thank A. Brown for his corrections.

\section{Appendix A: Dressed-state Hamiltonian}

\section{Hamiltonian of the system}

Quantization of the resonator eigenmodes results in the following expressions for the current and voltage operators and the Hamiltonian ${ }^{33}$

$$
\begin{aligned}
I(x) & =\sum \sqrt{\frac{\hbar \omega_{j}}{L_{\mathrm{r}}}}\left(a_{j}+a_{j}^{\dagger}\right) \cos k_{j} x, \\
V(x) & =-i \sum \sqrt{\frac{\hbar \omega_{j}}{C_{\mathrm{r}}}}\left(a_{j}-a_{j}^{\dagger}\right) \sin k_{j} x, \\
H_{\mathrm{r}} & =\sum \hbar \omega_{j} a_{j}^{\dagger} a_{j},
\end{aligned}
$$

where $k_{j}=\pi j / l, \omega_{j}=v k_{j}, v=1 / \sqrt{L C}$, and $a_{j}$ and $a_{j}^{\dagger}$ are the annihilation and creation operators for the photons of the $j$-th mode in the resonator. If the two signals (the probing one and the driving one) are close to the fundamental and the third harmonic components, we have the following for the relevant values:

the current at $x=0$ (which defines the inductive coupling to the qubit, see $H_{\text {int }}$ below)

$$
I(0)=I_{\mathrm{r} 0}\left[\left(a_{1}+a_{1}^{\dagger}\right)+\sqrt{3}\left(a_{3}+a_{3}^{\dagger}\right)\right], I_{\mathrm{r} 0}=\sqrt{\frac{\hbar \omega_{\mathrm{r}}}{L_{\mathrm{r}}}},
$$

the voltage at the boundaries (which defines coupling the resonator to the driving field, see $H_{\mu \mathrm{w}}$ below)

$$
V\left( \pm \frac{l}{2}\right)= \pm i V_{\mathrm{r} 0}\left[\left(a_{1}-a_{1}^{\dagger}\right)-\sqrt{3}\left(a_{3}-a_{3}^{\dagger}\right)\right], V_{\mathrm{r} 0}=\sqrt{\frac{\hbar \omega_{\mathrm{r}}}{C_{\mathrm{r}}}}
$$

and the resonator's Hamiltonian

$$
H_{\mathrm{r}}=\hbar \omega_{\mathrm{r}} a_{1}^{\dagger} a_{1}+3 \hbar \omega_{\mathrm{r}} a_{3}^{\dagger} a_{3} .
$$

The interaction between the two-mode resonator and the flux qubit is described with

$$
\begin{aligned}
H_{\mathrm{int}} & =M I(0) I_{\mathrm{qb}}=-\hbar\left[\mathrm{g}_{1}\left(a_{1}+a_{1}^{\dagger}\right)+\mathrm{g}_{3}\left(a_{3}+a_{3}^{\dagger}\right)\right] \tau_{z}, \\
\hbar \mathrm{g}_{1} & =M I_{\mathrm{r} 0} I_{\mathrm{p}}, \quad \mathrm{g}_{3}=\sqrt{3} \mathrm{~g}_{1},
\end{aligned}
$$

where $M$ is the mutual loop-resonator inductance.

The driving Hamiltonian originates from the energy of the left coupling capacitance $C_{0}, H_{C_{0}}=C_{0} \Delta V^{2}$. Here the probe + drive voltage to the left is the input voltage $V_{\text {in }}=$ $V_{\mathrm{p}} \sin \omega_{\mathrm{p}} t+V_{\mathrm{d}} \sin \omega_{\mathrm{d}} t$, which couples to the voltage in the resonator, $V(-l / 2)$. Then leaving only the slowly rotating terms, we obtain

$$
\begin{aligned}
H_{\mu \mathrm{w}} & =\xi_{\mathrm{p}}\left(a_{1} e^{i \omega_{\mathrm{p}} t}+a_{1}^{\dagger} e^{-i \omega_{\mathrm{p}} t}\right)+\xi_{\mathrm{d}}\left(a_{3} e^{i \omega_{\mathrm{d}} t}+a_{3}^{\dagger} e^{-i \omega_{\mathrm{d}} t}\right), \\
\xi_{\mathrm{p}} & =\frac{1}{2} C_{0} V_{\mathrm{p}} V_{\mathrm{r} 0}, \xi_{\mathrm{d}}=\frac{\sqrt{3}}{2} C_{0} V_{\mathrm{d}} V_{\mathrm{r} 0} .
\end{aligned}
$$

So, we have obtained the total Hamiltonian for the doublydriven qubit-resonator system (in which we rename the intracavity photon operators $a_{1} \equiv a$ and $a_{3} \equiv d$ ) :

$$
\begin{aligned}
H_{\mathrm{tot}}= & H_{\mathrm{qb}}+H_{\mathrm{r}}+H_{\mathrm{int}}+H_{\mu \mathrm{w}}, \\
H_{\mathrm{qb}}= & -\frac{\Delta}{2} \tau_{x}-\frac{\varepsilon_{0}}{2} \tau_{z}, \\
H_{\mathrm{r}}= & \hbar \omega_{\mathrm{r}} a^{\dagger} a+3 \hbar \omega_{\mathrm{r}} d^{\dagger} d, \\
H_{\mathrm{int}}= & -\hbar\left[\mathrm{g}_{1}\left(a+a^{\dagger}\right)+\mathrm{g}_{3}\left(d+d^{\dagger}\right)\right] \tau_{z}, \\
H_{\mu \mathrm{w}}= & \xi_{\mathrm{p}}\left(a e^{i \omega_{\mathrm{p}} t}+a^{\dagger} e^{-i \omega_{\mathrm{p}} t}\right)+ \\
& +\xi_{\mathrm{d}}\left(d e^{i \omega_{\mathrm{d}} t}+d^{\dagger} e^{-i \omega_{\mathrm{d}} t}\right) .
\end{aligned}
$$

\section{Reduced Hamiltonian}

Thus, we have the Hamiltonian for the qubit - two-mode resonator system. There, in respect to the experiment, we can assume the probe signal $\xi_{\mathrm{p}}$ to be relatively weak, so that $\langle n\rangle=$ $\left\langle a^{\dagger} a\right\rangle \ll 1$, while the driving signal is relatively strong, $\xi_{\mathrm{d}} \gg$ $\xi_{\mathrm{p}}$, so that $\langle N\rangle=\left\langle d^{\dagger} d\right\rangle \gg 1$. Observables are related to the former mode, and hence the latter mode can be traced out. In this way, one can obtain the reduced Hamiltonian, which describes the system of the one-mode resonator + "dressed" qubit. Then one can discuss the "dressed" relaxation as e.g. in Refs. [30 38], which approach allows to proceed analytically.

We now consider the Hamiltonian of the qubit + thirdharmonic driving in order to transform it to the dressed-state qubit Hamiltonian,

$$
\begin{aligned}
H_{\mathrm{qb}+\mathrm{d} .}= & -\frac{\Delta}{2} \tau_{x}-\frac{\varepsilon_{0}}{2} \tau_{z}+3 \hbar \omega_{\mathrm{r}} d^{\dagger} d+\quad \text { (A14) } \\
& +\hbar \mathrm{g}_{3}\left(d+d^{\dagger}\right) \tau_{z}+\xi_{\mathrm{d}}\left(d e^{i \omega_{\mathrm{d}} t}+d^{\dagger} e^{-i \omega_{\mathrm{d}} t}\right) .
\end{aligned}
$$

This does not include the probe signal (first harmonic), since all the operations in this subsection would not affect it.

First, let us eliminate the time dependence from the driving by applying the unitary operator $U_{1}=\exp \left(i \omega_{\mathrm{d}} t d^{\dagger} d\right)$. We obtain the new Hamiltonian

$$
\begin{aligned}
H_{1}= & U_{1} H_{\mathrm{qb}+\mathrm{d} .} U_{1}^{\dagger}+i \hbar \dot{U}_{1} U_{1}^{\dagger}= \\
= & -\frac{\Delta}{2} \tau_{x}-\frac{\varepsilon_{0}}{2} \tau_{z}+\hbar\left(3 \omega_{\mathrm{r}}-\omega_{\mathrm{d}}\right) d^{\dagger} d+ \\
& +\hbar \mathrm{g}_{3}\left(d e^{-i \omega_{\mathrm{d}} t}+d^{\dagger} e^{i \omega_{\mathrm{d}} t}\right) \tau_{z}+\xi_{\mathrm{d}}\left(d+d^{\dagger}\right) .
\end{aligned}
$$


For a large intracavity photon number $\langle N\rangle$, the driving field can be described as coherent state. Averaging the Hamiltonian with respect to the coherent state $|\alpha\rangle$ (note $d|\alpha\rangle=\alpha|\alpha\rangle$ ) yields,

$$
H_{2}=\left\langle H_{1}\right\rangle=\left\langle\alpha\left|H_{1}\right| \alpha\right\rangle=-\frac{\Delta}{2} \tau_{x}-\frac{\varepsilon_{0}+A_{\mathrm{d}} \cos \omega_{\mathrm{d}} t}{2} \tau_{z},
$$

where $A_{\mathrm{d}}=4 \alpha \hbar \mathrm{g}_{3}, \alpha=\sqrt{\langle N\rangle}$, and two constant terms $\hbar\left(3 \omega_{\mathrm{r}}-\omega_{\mathrm{d}}\right)\langle N\rangle$ and $2 \alpha \xi_{\mathrm{d}}$ have been omitted.

Next, we rewrite the Hamiltonian $H_{2}$ in the eigenbasis of its time-independent part, using the transformation $S=$ $\exp \left(i \varsigma \tau_{y} / 2\right)$ with $\tan \varsigma=-\Delta / \varepsilon_{0}$,

$$
H_{\mathrm{qb}}^{\prime}=-\frac{\Delta E}{2} \sigma_{z}-\frac{A_{\mathrm{d}} \cos \omega_{\mathrm{d}} t}{2}\left(\frac{\varepsilon_{0}}{\Delta E} \sigma_{z}-\frac{\Delta}{\Delta E} \sigma_{x}\right),
$$

which allows for the subsequent application of the rotatingwave approximation (RWA).

\section{RWA for weak driving}

For relatively weak driving $\left(A_{\mathrm{d}}<\hbar \omega_{\mathrm{d}}\right)$ we will use the conventional version of RWA. (Note that the conditions $A_{\mathrm{d}}\left\langle\hbar \omega_{\mathrm{d}}\right.$ and $\langle N\rangle \gg 1$ are consistent for weak coupling $\mathrm{g}_{3} \ll \omega_{\mathrm{d}}$.) For this we make the transformation with $U=\exp \left(-i \omega_{\mathrm{d}} t \sigma_{z} / 2\right)$, and omitting fast-rotating terms, obtain

$$
\widetilde{H}_{\mathrm{qb}}=U H_{\mathrm{qb}}^{\prime} U^{\dagger}+i \hbar \dot{U} U^{\dagger} \simeq-\frac{\widetilde{\varepsilon}}{2} \sigma_{z}+\frac{\widetilde{\Delta}}{2} \sigma_{x}
$$

where $\widetilde{\varepsilon}=\Delta E-\hbar \omega_{\mathrm{d}}, \widetilde{\Delta}=\Delta A_{\mathrm{d}} / 2 \Delta E$. Diagonalization of this Hamiltonian gives the dressed energy levels, Eq. (4). These transformations would also affect the interaction term, Eq. (A12): $\tau_{z} \rightarrow \frac{\varepsilon_{0}}{\Delta E} \sigma_{z}-\frac{\Delta}{\Delta E} \sigma_{x}$, where the second term can be neglected in RWA, while the first term results in the renormalization of the coupling coefficient: $\mathrm{g}_{1} \rightarrow \mathrm{g}_{\varepsilon}=\mathrm{g}_{1} \varepsilon_{0} / \Delta E$. Collecting all together, we obtain Hamiltonian (1). Then we move to the dressed-qubit eigenstates, using the transformation $\widetilde{S}=\exp \left(i \chi \sigma_{y} / 2\right)$ with $\tan \chi=\widetilde{\Delta} / \widetilde{\varepsilon}$, and get

$$
\begin{aligned}
\widetilde{H}^{\prime}= & \frac{\widetilde{\Delta E}}{2} \widetilde{\sigma}_{z}+\hbar \omega_{\mathrm{r}} a^{\dagger} a+\hbar \mathrm{g}_{\varepsilon}\left(a+a^{\dagger}\right) \times \\
& \times\left(\frac{\widetilde{\varepsilon}}{\widetilde{\Delta E}} \widetilde{\sigma}_{z}+\frac{\widetilde{\Delta}}{\widetilde{\Delta E}} \widetilde{\sigma}_{x}\right)+\xi_{\mathrm{p}}\left(a e^{i \omega_{\mathrm{p}} t}+a^{\dagger} e^{-i \omega_{\mathrm{p}} t}\right) .
\end{aligned}
$$

Then the second RWA is made with $U_{\mathrm{p}}=$ $\exp \left[i \omega_{\mathrm{p}} t\left(a^{\dagger} a+\widetilde{\sigma}_{z} / 2\right)\right]$, and, omitting the fast-rotating terms, we obtain Hamiltonian (11).

\section{RWA for strong driving}

To apply RWA to the Hamiltonian A17 in the limit of strong driving, we consider the following unitary transforma- tion: $W=\exp \left(-i \eta \sigma_{z} / 2\right)$, where $\eta$ is given by the integral from the second term in Eq. A17,

$$
\eta=\frac{\varepsilon_{0}}{\Delta E} \frac{A_{\mathrm{d}}}{\hbar \omega_{\mathrm{d}}} \sin \omega_{\mathrm{d}} t
$$

This results in the new Hamiltonian

$$
\begin{aligned}
H_{\mathrm{qb}}^{\prime \prime}= & -\frac{\Delta E}{2} \sigma_{z}+\widetilde{\Delta} \cos \omega_{\mathrm{d}} t\left(\sigma e^{i \eta}+\sigma^{\dagger} e^{-i \eta}\right)=(\mathrm{A} 21) \\
= & -\frac{\Delta E}{2} \sigma_{z}+\frac{\widetilde{\Delta}}{2}\left(e^{i \omega_{\mathrm{d}} t}+e^{-i \omega_{\mathrm{d}} t}\right) \times \\
& \times \sum_{l=-\infty}^{\infty} J_{l}\left(\frac{A_{\mathrm{d}}}{\hbar \omega_{\mathrm{d}}} \frac{\varepsilon_{0}}{\Delta E}\right)\left(\sigma e^{i l \omega_{\mathrm{d}} t}+\sigma^{\dagger} e^{-i l \omega_{\mathrm{d}} t}\right) .
\end{aligned}
$$

Then the RWA consists in applying another transformation, $W_{2}=\exp \left(-i k \omega_{\mathrm{d}} t \sigma_{z} / 2\right)$, and omitting the fast timedependent terms. We obtain in the vicinity of the $k$-th resonance, where $\Delta E-k \hbar \omega_{\mathrm{d}} \ll \Delta E$, the following

$$
\widetilde{H}_{\mathrm{qb}}^{(k)}=-\frac{\widetilde{\varepsilon}^{(k)}}{2} \sigma_{z}+\frac{\widetilde{\Delta}^{(k)}}{2} \sigma_{x}
$$

where

$$
\begin{aligned}
\widetilde{\varepsilon}^{(k)} & =\Delta E-k \hbar \omega_{\mathrm{d}}, \\
\widetilde{\Delta}^{(k)} & =\Delta \frac{k \hbar \omega_{\mathrm{d}}}{\varepsilon_{0}} J_{k}\left(\frac{A_{\mathrm{d}}}{\hbar \omega_{\mathrm{d}}} \frac{\varepsilon_{0}}{\Delta E}\right) .
\end{aligned}
$$

Then for the dressed energy distance we obtain $\widetilde{\Delta E}^{(k)}=$ $\sqrt{\widetilde{\varepsilon}^{(k) 2}+\widetilde{\Delta}^{(k) 2}}$ or explicitly 9,44

$$
\widetilde{\Delta E}^{(k)}=\sqrt{\left(\Delta E-k \hbar \omega_{\mathrm{d}}\right)^{2}+\left(\Delta \frac{k \hbar \omega_{\mathrm{d}}}{\varepsilon_{0}} J_{k}\left(\frac{A_{\mathrm{d}}}{\hbar \omega_{\mathrm{d}}} \frac{\varepsilon_{0}}{\Delta E}\right)\right)^{2}} .
$$

In particular, at weak driving only the levels with $k=1$ are relevant and $J_{1}(x) \approx x / 2$; then $\widetilde{\Delta}^{(1)} \approx \widetilde{\Delta}$ and $\widetilde{\Delta E}^{(1)}$ coincides with $\widehat{\Delta E}$, obtained in the weak-driving limit, Eq. (4). Moreover, in the limit $\varepsilon_{0} \gg \Delta$ this gives

$$
\widetilde{\Delta E}^{(k)}=\sqrt{\left(\varepsilon_{0}-k \hbar \omega_{\mathrm{d}}\right)^{2}+\left(\Delta J_{k}\left(A_{\mathrm{d}} / \hbar \omega_{\mathrm{d}}\right)\right)^{2}},
$$

which is in agreement with the results of RWA used, e.g., in Refs. [38. 42].

Applying the above transformations would also affect the qubit-resonator interaction term. As the result we obtain the total Hamiltonian in the RWA, given by Eq. (1) with the substitutions: $\widetilde{\varepsilon} \rightarrow \widetilde{\varepsilon}^{(k)}$ and $\widetilde{\Delta} \rightarrow \widetilde{\Delta}^{(k)}$. 
1 W.D. Oliver, Y. Yu, J.C. Lee, K.K. Berggren, L.S. Levitov, and T.P. Orlando, Science 310, 1653 (2005).

${ }^{2}$ V.I. Shnyrkov, Th. Wagner, D. Born, S.N. Shevchenko, W. Krech, A.N. Omelyanchouk, E. Il'ichev, and H.-G. Meyer, Phys. Rev. B 73, 024506 (2006).

${ }^{3}$ C.M. Wilson, T. Duty, F. Persson, M. Sandberg, G. Johansson, and P. Delsing, Phys. Rev. Lett. 98, 257003 (2007).

${ }^{4}$ B.R. Mollow, Phys. Rev. A 5, 2217 (1972).

5 F.Y. Wu, S. Ezekiel, M. Ducloy, and B.R. Mollow, Phys. Rev. Lett. 38, 1077 (1977).

6 J. Zakrzewski, M. Lewenstein, and T.W. Mossberg, Phys. Rev. A 44, 7717 (1991).

7 A. Wallraff, D.I. Schuster, A. Blais, J.M. Gambetta, J. Schreier, L. Frunzio, M.H. Devoret, S.M. Girvin, and R.J. Schoelkopf, Phys. Rev. Lett. 99, 050501 (2007).

8 A. Papageorge, A. Majumdar, E.D. Kim, and J. Vučković, New J. Phys. 14, 013028 (2012).

9 M. Silveri, J. Tuorila, M. Kemppainen, and E. Thuneberg, Phys. Rev. B 87, 134505 (2013).

${ }^{10}$ G.M. Reuther, D. Zueco, F. Deppe, E. Hoffmann, E.P. Menzel, T. Weiß1, M. Mariantoni, S. Kohler, A. Marx, E. Solano, R. Gross, and P. Hänggi, Phys. Rev. B 81, 144510 (2010).

11 J. Zhao, Y. Yu, and B.-B. Jin, IEEE Trans. Appl. Supercond. 23, 1701705 (2013).

${ }^{12}$ L.S. Bishop, J.M. Chow, J. Koch, A.A. Houck, M.H. Devoret, E. Thuneberg, S.M. Girvin, and R. J. Schoelkopf, Nature Phys. 5, 105 (2009).

13 J.M. Fink, M. Baur, R. Bianchetti, S. Filipp, M. Göppl, P.J. Leek, L. Steffen, A. Blais, and A. Wallraff, Phys. Scr. T137, 014013 (2009).

${ }^{14}$ F. Hocke, X. Zhou, A. Schliesser, T. Kippenberg, H. Huebl, and R. Gross, New J. Phys. 14, 123037 (2012).

15 C. Cohen-Tannoudji, J. Dupont-Rock, and G. Grynberg, AtomPhoton Interactions. Basic Processes and Applications, John Wiley, New York (1998), Chap. 6.

16 Y. Nakamura, Yu.A. Pashkin, and J.S. Tsai, Phys. Rev. Lett. 87, 246601 (2001)

17 Y.X. Liu, C.P. Sun, and F. Nori, Phys. Rev. A 74, 052321 (2006).

18 G. Sun, X. Wen, B. Mao, Y. Yu, J. Chen, W. Xu, L. Kang, P. Wu, and S. Han, Phys. Rev. B 83, 180507(R) (2011).

19 M. Yan, E.G. Rickey, and Y. Zhu, Phys. Rev. A 64, 013412 (2001).

${ }^{20}$ C.L. Garrido Alzar, H. Perrin, B.M. Garraway, and V. Lorent, Phys. Rev. A 74, 053413 (2006).

21 A.P. Saiko and R. Fedaruk, JETP Lett. 91, 681 (2010).

22 A.M. Satanin, M.V. Denisenko, A.I. Gelman, and F. Nori, arXiv: 1305.4800 (2013).

23 G.A. Abovyan and G.Yu. Kryuchkyan, Phys. Rev. A 88, 033811 (2013).

${ }^{24}$ O.V. Astafiev, A.A. Abdumalikov, Jr., A.M. Zagoskin, Yu.A.
Pashkin, Y. Nakamura, and J.S. Tsai, Phys. Rev. Lett. 104, 183603 (2010).

${ }^{25}$ G. Oelsner, P. Macha, O.V. Astafiev, E. Il'ichev, M. Grajcar, U. Hübner, B.I. Ivanov, P. Neilinger, and H.-G. Meyer, Phys. Rev. Lett. 110, 053602 (2013).

${ }^{26}$ K. Koshino, H. Terai, K. Inomata, T. Yamamoto, W. Qiu, Z. Wang, and Y. Nakamura, Phys. Rev. Lett. 110, 263601 (2013).

27 Ya.S. Greenberg, A. Izmalkov, M. Grajcar, E. Il'ichev, W. Krech, and H.-G. Meyer, Phys. Rev. B 66, 224511 (2002).

28 Ya.S. Greenberg, E. Il'ichev, and A. Izmalkov, Europhys. Lett. 72, 880 (2005).

${ }^{29}$ Ya.S. Greenberg, Phys. Rev. B 76, 104520 (2007).

30 J. Hauss, A. Fedorov, S. André, V. Brosco, C. Hutter, R. Kothari, S. Yeshwanth, A. Shnirman, and G. Schön, New J. Phys. 10, 095018 (2008).

31 A.E. Mefed and V.A. Atsarkin, Pis'ma Zh. Eksp. Teor. Fiz. 25, 233 (1977) [JETP Lett. 25, 215 (1977)].

32 G. Oelsner, S.H.W. van der Ploeg, P. Macha, U. Hübner, D. Born, S. Anders, E. Il'ichev, H.-G. Meyer, M. Grajcar, S. Wünsch, M. Siegel, A.N. Omelyanchouk, and O. Astafiev, Phys. Rev. B 81, 172505 (2010).

33 A.N. Omelyanchouk, S.N. Shevchenko, Ya.S. Greenberg, O. Astafiev and E. Il'ichev, Low Temp. Phys. 36, 893 (2010).

34 J. Tuorila, M. Silveri, M. Sillanpää, E. Thuneberg, Y. Makhlin, and P. Hakonen, Phys. Rev. Lett. 105, 257003 (2010).

35 A.D. Greentree, C. Wei, and N.B. Manson, Phys. Rev. A 59, 4083 (1999).

36 M.O. Scully and M.S. Zubairy, Quantum Optics (Cambridge, Cambridge University Press) (1997).

37 H. Ian, Y.X. Liu, and F. Nori, Phys. Rev. A 81, 063823 (2010).

38 C.M. Wilson, G. Johansson, T. Duty, F. Persson, M. Sandberg, and P. Delsing, Phys. Rev. B 81, 024520 (2010).

39 Y. Mu and C.M. Savage, Phys. Rev. A 46, 5944 (1992).

${ }^{40}$ S. Ashhab, J.R. Johansson, A.M. Zagoskin, and F. Nori, New J. Phys. 11, 023030 (2009).

${ }^{41}$ L. Ella and E. Buks, arXiv:1210.6902

${ }^{42}$ S.N. Shevchenko, S. Ashhab, and F. Nori, Phys. Rev. B 85, 094502 (2012).

43 J. Li, M.P. Silveri, K.S. Kumar, J.-M. Pirkkalainen, A. Vepsäläinen, W.C. Chien, J. Tuorila, M.A. Sillanpää, P.J. Hakonen, E.V. Thuneberg, and G. S. Paraoanu, Nat. Commun. 4, 1420 (2013).

${ }^{44}$ W. Krech, D. Born, V. Shnyrkov, T. Wagner, M. Grajcar, E. Il'ichev, H.-G. Meyer, and Y. Greenberg, IEEE Trans. Appl. Supercond. 15, 876 (2005).

45 It is necessary to distinguish the number of photons $n$ from the average photon number $\langle n\rangle=\sum n\langle n|\rho| n\rangle$. The latter, while being small, contains contributions from many $n$ states. 